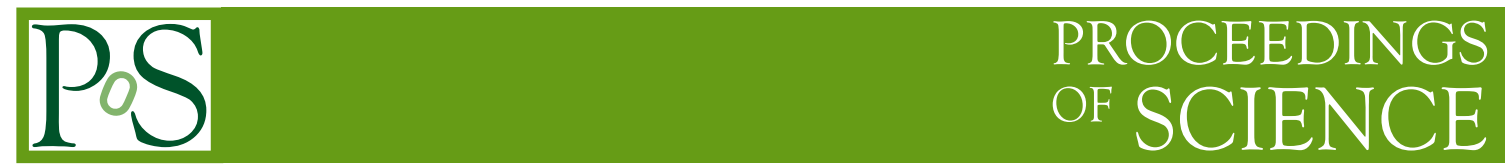

\title{
Nikos Antoniou memorial
}

\section{Fotis K. Diakonos ${ }^{a}$ and Costas G. Papadopoulos ${ }^{b}$}

${ }^{a}$ Faculty of Physics, University of Athens, GR-15784 Athens, Greece

${ }^{b}$ Institute of Nuclear and Particle Physics, NCSR Demokritos, Agia Paraskevi, 15310, Greece

E-mail: fdiakono@phys.uoa.gr, costas.papadopoulos@cern.ch

19TH HELLENIC SCHOOL AND WORKSHOPS ON ELEMENTARY PARTICLE PHYSICS AND GRAVITY, August 31 - September 25, 2019 Corfu, Greece 


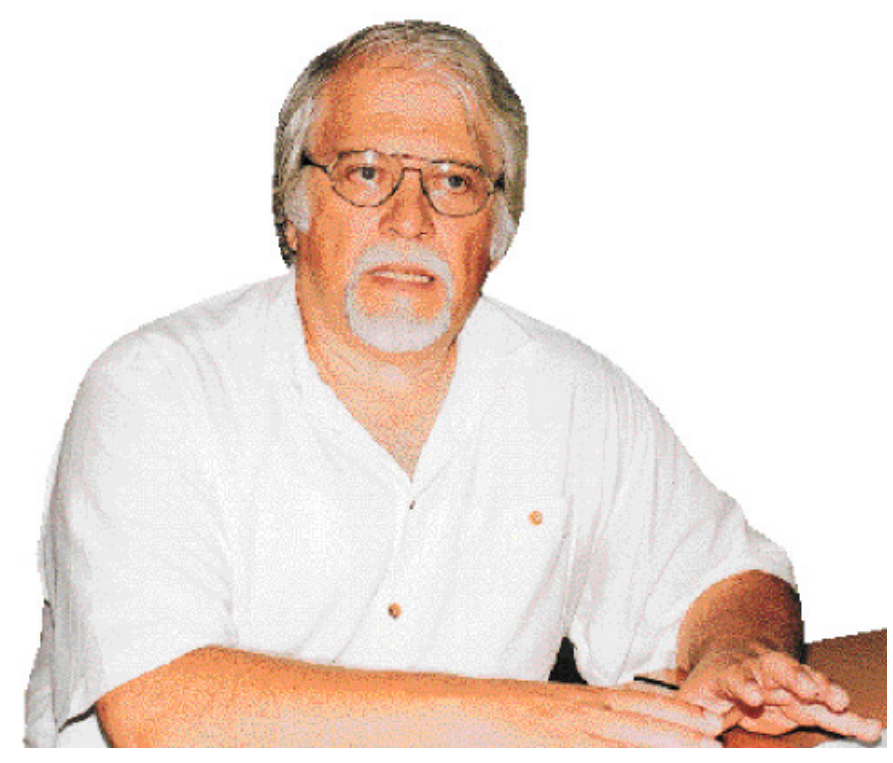

Nikos Antoniou (1939 - 2020)

Nikos Antoniou, who passed away suddenly on February 13th, 2020, was undoubtedly a dominant personality in the community of Theoretical Particle Physics in Greece. This is reflected also in the multitude of science-related administrative responsibilities overtaken by him during his career: He was member of the Board of Directors in the Hellenic Atomic Energy Commission during the years 1974-1977, president of the Hellenic Physical Society for the years 1981-1983, chairman of the Greek National Advisory Committee for CERN from 1982 to 1986, scientific director of the National Research Center "Demokritos" for seven years (1982-1989), scientific delegate of Greece to the CERN Council in the same time interval (1982-1989), to name a few examples.

Born in 1939, he was a graduate of the National Technical University of Athens and he received his doctorate in 1967 from the University of Birmingham. He held research positions at the University of Oxford, the ICTP in Trieste, the University of Tübingen, the Technion, CERN, the University of Cyprus, the University of Santiago de Compostela and the National Center for Scientific Research "Demokritos". He joined the faculty of the Physics Department of the National and Kapodistrian University of Athens in 1975, from which he retired in 2006 with the status of Professor Emeritus.

Focusing on his work as a researcher, and we think that this is the way he would like us remember him, Nikos Antoniou realized very early the importance, beauty and richness of the phase diagram of strongly interacting matter. Already before the study of the QCD phase diagram became the focus of extensive theoretical work in Lattice QCD and in effective models, he recognized the role of intermittent patterns in multiparticle production processes as a signature of cooperative phenomena in strongly interacting systems. His scientific life was dedicated to reveal the connection of such patterns with the critical behaviour of strongly interacting matter, using intermittency as the magnifying lens. He was one of the first theoreticians worldwide who introduced the concepts of fractal geometry and complexity in Theoretical Particle Physics and particularly in studying particle production from fireballs created in collisions of nuclei at ultra-relativistic energies. He always insisted that in order to detect and correctly describe critical fluctuations in hot and dense 
QCD matter one should search for fluctuations of quantities which could play the role of an order parameter. Additionally he was proponent of the view that the crucial factor for the detection of the QCD critical point, the holly grail of the contemporary strong interaction physics, was the achievement of the appropriate vacuum excitation. Thus, he had the revolutionary idea that even relatively small nuclei colliding with the appropriate energy could produce a fireball at the critical state. This was in full contradiction with the existing common belief that critical behaviour is only achievable through the collision of very large nuclei. A great success of his scientific efforts was the observation of critical fluctuations in collisions of silicon ions at the NA49 experiment in SPS, CERN. This unexpected finding initiated the "Light Ion Program" realized by the latest generation colliding ion experiment NA61/SHINE at CERN, SPS.

As a person Nikos Antoniou was enthusiastic and he was able to transfer this enthusiasm to his friends, colleagues and collaborators. His word was always very precise, deep and strict at the same time. He was very substantial and simple. He was always defending strongly the scientific ethics in research and he consistently resisted to all attempts of commercialization and subordination of science. Passing away Nikos Antoniou left an irreplaceable void. We will always remember him as an inspiring teacher, an invaluable collaborator and most importantly for his free scientific mind. 